\title{
Thyroid disease and vascular risk
}

\author{
Authors: Avais Jabbar ${ }^{A}$ and Salman Razvi ${ }^{B}$
}

Subclinical hypothyroidism ( $\mathrm{SCH}$ ) is a common condition seen in up to $10 \%$ of adults, mainly women and the elderly. Several prospective longitudinal cohort studies have shown a higher risk of cardiovascular disease in people with SCH but mainly in younger individuals. There are also a number of interventional trials that have shown that treatment of $\mathrm{SCH}$ with levothyroxine improves cardiovascular risk factors, but there is a dearth of level 1 evidence regarding cardiovascular events. In addition, there is increasing proof concerning the association of abnormal thyroid function at the time of an acute myocardial infarction with adverse cardiovascular outcomes. This review describes the literature dealing with thyroid function in relation to cardiovascular disease and also outlines the effect of treatment in addressing cardiovascular risk.

KEYWORDS: Subclinical hypothyroidism, cardiovascular disease, acute myocardial infarction

\section{Introduction}

Subclinical hypothyroidism (SCH) is diagnosed when serum thyroid stimulating hormone (TSH) levels are elevated but serum thyroid hormone concentrations are normal. The prevalence of $\mathrm{SCH}$ is higher than that of type 2 diabetes mellitus (5-10\%) and is more common in women and the elderly. ${ }^{1}$ However, despite its high prevalence, the clinical implications of $\mathrm{SCH}$ are much debated. Therefore there is a difference of opinion among clinicians regarding population screening and treatment. SCH has been associated with increased cardiovascular morbidity and mortality, especially in younger individuals and much recent research has focused on the implications $\mathrm{SCH}$ has on cardiovascular disease. Although $\mathrm{SCH}$ is associated with minor dyslipidaemia, this is not sufficient to account for the increased vascular risk seen. Other underlying mechanisms such as increased systemic vascular resistance, central arterial stiffness, vascular endothelial dysfunction, haemostatic and platelet dysfunction have all been demonstrated in $\mathrm{SCH} .{ }^{2}$ The purpose of this review is to outline the different mechanisms by which $\mathrm{SCH}$ may lead

Authors: ${ }^{A}$ clinical research associate, Newcastle University, Newcastleupon-Tyne, UK; ${ }^{B}$ consultant endocrinologist, Department of Endocrinology, Queen Elizabeth Hospital, Gateshead, UK, and honorary senior lecturer, Newcastle University, Newcastle-upon-Tyne, UK to cardiovascular disease and to provide an overview of the evidence we have so far. We will also review the benefits of treating $\mathrm{SCH}$ based on current literature.

\section{Mechanisms underlying the increased cardiovascular risk in $\mathrm{SCH}$}

Thyroid hormones have a direct impact on cardiovascular haemodynamics. The most common effects of an underactive thyroid state include diastolic dysfunction, a decrease in cardiac preload, systolic dysfunction and an increased systemic vascular resistance (SVR) which increases afterload. To understand this, we first have to understand the intranuclear activity of thyroid hormones on the myocyte. Such activity includes the activation of triiodothyronine (T3) genes within the nucleus which encode for both functional and regulatory proteins. Examples of such proteins include the two myosin heavy chains (alpha and beta), phospholamban, sarcoplasmic reticulum proteins and calciumactivated ATPase ( $\mathrm{Ca}^{2+}$-ATPase). The two myosin heavy chains form an important component of the cardiac myocyte contractile apparatus whereas both phospholambin and $\mathrm{Ca}^{2+}$-ATPase regulate release and reuptake of calcium from the sarcoplasmic reticulum. ${ }^{3}$ It is this regulation of calcium that is a key regulatory component in systolic function and diastolic relaxation, which both become impaired by an underactive thyroid state.

In addition, thyroid hormones are involved in lipid metabolism. The association between hypothyroidism and hypercholesterolemia and elevated low-density lipoproteins (LDLs) has been known for many years, with some estimates showing a link present in up to $90 \%$ of cases. ${ }^{4}$ Furthermore, elevated levels of lipids are also evident in patients with $\mathrm{SCH}$, suggesting an increased risk for atherosclerosis; however, the evidence remains controversial based on different populationbased studies. ${ }^{2}$ In some studies, such as the Whickham Survey and National Health and Nutritional Examination Survey (NHANES) III, there was no link between SCH and hyperlipidaemia, whereas other studies showed a link as well as a relative increase in serum lipids with an increase in serum $\mathrm{TSH}$ in $\mathrm{SCH} .{ }^{2,5}$ Hyperlipidaemia in an underactive thyroid state is due to a decrease in LDL receptors, which reduces the clearance of cholesterol from the liver, and decreased activity of the enzyme cholesterol $7 \alpha$-hydroxylase, which is usually activated by thyroid hormones in breaking down cholesterol. ${ }^{4}$

It is already known that overt hypothyroidism can cause left ventricular diastolic dysfunction by reducing the activity of $\mathrm{Ca}^{2+}$-ATPase within the sarcoplasmic reticulum, which leads to reduced reuptake of calcium during diastole; this in turn 
leads to impaired ventricular relaxation ${ }^{3}$ which impairs left ventricular filling during diastole (preload). Similar findings also occur in SCH patients, with findings showing a more prolonged isovolumetric relaxation time and impaired peak filling rate, which are key components to diastolic function. Diastolic heart failure has implications in $\mathrm{SCH}$ patients due to diastolic heart failure being common in the elderly and the fact that it likely reduces exercise tolerance in younger patients. ${ }^{6}$ The main cause of systolic impairment in subclinical hypothyroidism is likely to be reduced inotropic effects on the heart as well as increased SVR, which increases the afterload. The increase in SVR can be explained by reduced tissue metabolism and thermogenesis, as well as impaired smooth muscle relaxation, which is again linked to impaired calcium reuptake. ${ }^{7}$ Cardiac magnetic resonance imaging (MRI) has already demonstrated how $\mathrm{SCH}$ causes diastolic dysfunction, reduces cardiac preload and afterload with such findings normalising after levothyroxine treatment. ${ }^{8}$

$\mathrm{SCH}$ can also cause cardiovascular disease via systemic hypertension and impaired vascular function. The causes of systemic hypertension in SCH are an increase in SVR and endothelial dysfunction, as well as increased arterial stiffness. Increased arterial stiffness is also a precursor to atherosclerosis. Pulse wave velocity, a measure of arterial stiffness, has been shown to be increased in $\mathrm{SCH}$, whereas advanced imaging of the brachial artery, which is a measure of endothelial function, has shown that vasodilation of the endothelium is impaired in SCH patients, with such changes improving after levothyroxine treatment. ${ }^{9}$ Randomised controlled studies have shown that levothyroxine significantly lowers LDL cholesterol levels and improves endothelial function in comparison to placebo in patients with SCH. ${ }^{10}$ Numerous factors are likely to contribute to endothelial dysfunction in SCH. Hyperlipidaemia is likely to be one cause due to its role in atherosclerosis. Thyroid autoantibodies in $\mathrm{SCH}$ also play a key role in endothelial dysfunction, with evidence showing that patients with $\mathrm{SCH}$ and thyroid antibodies have increased endothelial dysfunction than those who have SCH alone. ${ }^{11,12}$ The fact that vascular adhesive molecules such as E-selectin and P-selectin are increased in Hashimoto's thyroiditis indicates that thyroid antibodies have a key role in inflammation. ${ }^{13}$ Both hyperlipaemia and thyroid antibodies are thought to reduce the expression of endothelial nitric oxide synthase and therefore impair the ability of the artery to vasodilate..$^{11,12}$

Alterations in coagulation parameters in $\mathrm{SCH}$ might play a role in the potential development of atherosclerosis. In one study comparing women with SCH with euthyroid controls, factor VII activity and the factor VII activity:factor VII antigen ratio were significantly increased in women with $\mathrm{SCH}$, whereas there were no differences in von Willebrand factor or other haemostatic factors tested. ${ }^{14}$ In another study, decreased antithrombin III activity and increased levels of fibrinogen, factor VII and plasminogen activator inhibitor antigen were found in $\mathrm{SCH}$ patients. ${ }^{15}$ A recent study using the Badimon chamber, a model which simulates ex vivo coronary artery blood flow through a diseased artery, has shown that thrombus area in patients with SCH 7-10 days post non-ST elevation myocardial infarction is larger than in euthyroid patients, despite the use of aspirin and clopidogrel..$^{16}$ This may help explain the higher cardiovascular risk seen in patients with $\mathrm{SCH}$, as such a state is likely to be thrombogenic.

\section{Clinical outcomes of SCH in cardiovascular disease}

A number of observational studies have shown that individuals with abnormal thyroid function have adverse outcomes including higher mortality. Even minor changes in thyroid hormone concentration may impact adversely on the cardiovascular system. Mild hypothyroidism ( $\mathrm{SCH}$ ) has been associated with a $20-80 \%$ increased vascular morbidity and mortality risk, with a study by Parle et al showing how a single measurement of low TSH in individuals aged above 60 years was associated with increased mortality from cardiovascular diseases, whereas another study has shown that $\mathrm{SCH}$ is a strong independent risk factor for coronary heart disease. ${ }^{17,18}$ The Rotterdam Study showed that middle-aged women with $\mathrm{SCH}$ were more likely to have a myocardial infarction and calcification of the aorta. ${ }^{19}$ These findings are supported by a meta-analysis which shows that subclinical hypothyroidism is associated with an increased risk of coronary heart disease. ${ }^{20}$ Not all prospective cohort studies have shown a link between SCH and cardiovascular disease. ${ }^{21}$ Recently, a patient-level meta-analysis of 55,287 participants from 11 prospective cohort studies showed that $\mathrm{SCH}$ was associated with coronary heart disease and mortality in those with higher TSH levels and that only a mild increase in TSH was not associated with CHD. ${ }^{22}$ However, most cohort studies and meta-analyses have not taken into account subsequent treatment with thyroid hormones, therefore their results may have missed an important confounder.

\section{Thyroid function in acute myocardial infarction}

Although prospective observational cohort studies largely show that $\mathrm{SCH}$ has a worse cardiovascular outcome, $\mathrm{SCH}$ in acute myocardial infarction (AMI) is also likely to be detrimental to cardiovascular health. SCH after admission for an acute cardiac problem has been associated with an up to 3.6 fold increase in cardiac mortality and a 2.3 fold increase in overall death. ${ }^{23}$ It is likely that $\mathrm{SCH}$ around the time of AMI is maladaptive rather than adaptive and that such a state is not a consequence of stress, but rather a permissive state that favours cardiac failure. AMI leads to lower serum thyroid hormones as well as downregulation of thyroid hormone receptors in the myocardium, leading to tissue hypothyroidism. A prospective study by Friberg et al showed that thyroid hormone levels rapidly decline within a week after AMI. ${ }^{24}$ Furthermore, another study by Friberg et al has shown that in-hospital and post discharge mortality is higher in patients with the most suppressed thyroid hormone levels post AMI, indicating that a suppressed thyroid state is associated with a worse prognosis. ${ }^{25}$ In comparison to other organs, the heart is more vulnerable to a hypothyroid state due to cardiac myocytes having no capability in converting the precursor $\mathrm{T} 4$ to $\mathrm{T} 3$ which can occur in other organs. $^{7}$ This can explain why a SCH state may make the myocardium relatively hypothyroid despite having no effect on a patient's overall thyroid state.

Thyroid hormones have a direct role in post ischaemic cardiac remodelling by effects on angiogenesis, cell repair and metabolism. Furthermore, thyroid hormones can have an effect on cardiac remodelling by inhibiting cardiac myocyte death pathways, preventing hypertrophy, improving myocardial perfusion and reducing fibrous tissue from forming. Myocyte apoptosis plays a key part in myocyte death after AMI. The 
protein kinase $\mathrm{B}$ (PKB) signalling pathway is integral in protecting against apoptosis via phosphorylation of different substrates and $\mathrm{T} 3$ has been shown to activate the PKB pathway in the area affected by myocardial infarction. In a rat heart model of ischaemia reperfusion injury, the administration of T3 during the reperfusion period reduced myocyte apoptosis via the PKB pathway and this helped recovery significantly. ${ }^{26}$

Apoptosis and myocyte protection is also regulated by mitochondrial function. The cardioprotective mechanisms of mitochondria include generating an antioxidant response and controlling the calcium flux into the myocardium. Mitochondrial dysfunction plays a key role in the progression of heart failure indicating that preserving mitochondrial function is vital. Thyroid hormones are vital for mitochondrial function as shown from a study in which $\mathrm{T} 3$ administration rescued mitochondrial function and therefore prevented cardiac remodelling and reduced myocyte apoptosis in a post-ischaemic rat model. ${ }^{27}$

\section{Subclinical hypothyroidism treatment with levothyroxine}

Numerous studies have shown a beneficial effect of levothyroxine on cardiovascular risk factors in $\mathrm{SCH}$. Randomised control studies are needed to show a beneficial effect of treatment in $\mathrm{SCH}$ with regard to cardiovascular outcomes. Analysis of more than 3,000 participants aged 65 years or more without heart failure at baseline, who were followed up for 12 years in the Cardiovascular Health Study, showed that SCH participants who were treated with levothyroxine had a $72 \%$ reduction in heart failure events. ${ }^{28}$ A retrospective study of patients from the United Kingdom General Practitioner Research Database showed that treatment with levothyroxine in $\mathrm{SCH}$ was associated with fewer ischaemic heart disease events in younger individuals, but this was not evident in older people. ${ }^{29}$

Other studies have shown a beneficial effect of levothyroxine therapy on cardiovascular risk factors such as cholesterol, endothelial function and carotid intima-media thickness. ${ }^{10,30}$ Additionally, studies have also shown how $\mathrm{SCH}$ affects cardiac pump function. SCH has been shown to cause diastolic dysfunction, decrease cardiac preload and increase cardiac afterload with cardiac MRI with such changes normalising after levothyroxine therapy. ${ }^{8}$ Low levels of thyroid hormones have been observed in heart failure that are directly proportional to its severity, with beneficial outcomes being noted with thyroid hormone replacement. In a prospective study of 112 patients with New York Heart Association (NYHA) class 2-4 heart failure, $15 \%$ of patients had either hypothyroidism or $\mathrm{SCH}$ for which they were taking thyroxine replacement, whereas $31 \%$ had low T3 levels. ${ }^{31}$ Other studies have shown how levothyroxine replacement in such patients increases cardiac output, exercise performance and cardiac index without any evidence for tachyarrythmias or myocardial ischaemia. ${ }^{32,33}$

The current guidelines recommend treatment of $\mathrm{SCH}$ in younger patients and in older patients when $\mathrm{TSH}>10$ and in those who are symptomatic. ${ }^{34}$ Despite known cardiovascular risks of $\mathrm{SCH}$, there is not enough evidence for treating all patients at present due to current data being mostly based on observational studies or from small interventional trials with cardiac risk factor change as outcomes. For this reason randomised controlled trials are needed to evaluate the benefits of treatment of $\mathrm{SCH}$ in reducing cardiovascular risk. In the meantime, it may be beneficial to treat $\mathrm{SCH}$ patients who are at high risk of cardiovascular disease or those that may have evidence of cardiac impairment. Current data suggests that treatment of $\mathrm{SCH}$ is likely to be more beneficial in younger patients than the elderly; however, once again more studies are needed to ascertain the risks or benefits of levothyroxine treatment in the elderly with SCH. Further studies are needed to assess the outcomes of patients with $\mathrm{SCH}$ post myocardial infarction.

\section{Acknowledgements}

Both AJ and SR are funded by NIHR (CDF-2012-05-231). This report is independent research supported by the National Institute for Health Research Career Development Fellowship CDF-2012-05-231. The views expressed in this publication are those of the authors and not necessarily those of the NHS, the National Institute for Health Research or the Department of Health.

\section{References}

1 Tunbridge WM, Evered DC, Hall R. The spectrum of thyroid disease in a community: the Whickham survey. Clin Endocrinol 1977;7:481-93.

2 Biondi B, Cooper DS. The clinical significance of subclinical thyroid dysfunction. Endocr Rev 2008;29:76-131.

3 Kahaly GJ, Dillmann WH. Thyroid hormone action in the heart. Endocr Rev 2005;26:704-28.

4 Duntas LH. Thyroid disease and lipids. Thyroid 2002;12:287-93.

5 Asvold BO, Vatten LJ, Nilsen TI et al. The association between TSH within the reference range and serum lipid concentrations in a population-based study. The HUNT Study. Eur J Endocrinol 2007;156:181-6.

6 Aghini-Lombardi F, Di Bello V, Talini E et al. Early textural and functional alterations of left ventricular myocardium in mild hypothyroidism. Eur J Endocrinol 2006;155:3-9.

7 Klein I, Danzi S. Thyroid disease and the heart. Circulation 2007;116:1725-35.

8 Ripoli A, Pingitore A, Favilli B et al. Does subclinical hypothyroidism affect cardiac pump performance? Evidence from a magnetic resonance imaging study. J Am Coll Cardiol 2005;45:439-45.

9 Nagasaki T, Inaba M, Yamada S et al. Changes in brachial-ankle pulse wave velocity in subclinical hypothyroidism during normalization of thyroid function. Biomed Pharmacother 2007;61:482-7.

10 Razvi S, Ingoe L, Keeka G et al. The beneficial effect of L-thyroxine on cardiovascular risk factors, endothelial function, and quality of life in subclinical hypothyroidism: randomized, crossover trial. J Clin Endocrinol Metab 2007;92:1715-23.

11 Cikim AS, Oflaz H, Ozbey N et al. Evaluation of endothelial function in subclinical hypothyroidism and subclinical hyperthyroidism. Thyroid 2004;14:605-9.

12 Türemen EE, Cetinarslan B, Sahin T et al. Endothelial dysfunction and low grade chronic inflammation in subclinical hypothyroidism due to autoimmune thyroiditis. Endocr J 2011;58:349-54.

13 Marazuela M, Sanchez-Madrid F, Acevedo A et al. Expression of vascular adhesion molecules on human endothelia in autoimmune thyroid disorders. Clin Exp Immunol 1995;102:328-34.

14 Müller B, Tsakiris DA, Roth CB et al. Haemostatic profile in hypothyroidism as potential risk factor for vascular or thrombotic disease. Eur J Clin Invest 2001;31:131-7.

15 Cantürk Z, Cetinarslan B, Tarkun I et al. Hemostatic system as a risk factor for cardiovascular disease in women with subclinical hypothyroidism. Thyroid 2003;13:971-7. 
16 Viswanathan G, Balasubramaniam K, Hardy R et al. Blood thrombogenicity is independently associated with serum TSH levels in post non ST elevation acute coronary syndrome. J Clin Endocrinol Metab 2014:jc20133062.

17 Parle JV, Maisonneuve P, Sheppard MC et al. Prediction of allcause and cardiovascular mortality in elderly people from one low serum thyrotropin result: a 10-year cohort study. Lancet 2001;358:861-5.

18 Walsh JP, Bremner AP, Bulsara MK et al. Subclinical thyroid dysfunction as a risk factor for cardiovascular disease. Arch Intern Med 2005;165:2467-72.

19 Hak AE, Pols HAP, Visser TJl et al. Subclinical hypothyroidism is an independent risk factor for atherosclerosis and myocardial infarction in elderly women: the Rotterdam Study. Ann Intern Med 2000;132:270-8.

20 Rodondi N, Aujesky D, Vittinghoff E et al. Subclinical hypothyroidism and the risk of coronary heart disease: a meta-analysis. Am J Med 2006;119:541-51.

21 Cappola AR, Fried LP, Arnold AM et al. Thyroid status, cardiovascular risk, and mortality in older adults. JAMA 2006;295:1033-41.

22 Rodondi N, den Elzen WP, Bauer DC et al. Thyroid Studies Collaboration. Subclinical hypothyroidism and the risk of coronary heart disease and mortality. JAMA 2010;304:1365-74.

23 Molinaro S, Iervasi G, Lorenzoni V et al. Persistence of mortality risk in patients with acute cardiac diseases and mild thyroid dysfunction. Am J Med Sci 2012;343:65-70.

24 Friberg L, Werner S, Eggertsen G et al. Rapid down-regulation of thyroid hormones in acute myocardial infarction: is it cardioprotective in patients with angina? Arch Intern Med 2002;162:1388-94.

25 Friberg L, Drvota V, Bjelak et al. Association between increased levels of reverse triiodothyronine and mortality after acute myocardial infarction. Am J Med 2001;111:699-703.
26 Pantos C, Mourouzis I, Saranteas T et al. Thyroid hormone improves postischaemic recovery of function while limiting apoptosis: a new therapeutic approach to support hemodynamics in the setting of ischaemia-reperfusion? Basic Res Cardiol 2009;104:69-77.

27 Forini F, Lionetti V, Ardehali H et al. Early long-term L-T3 replacement rescues mitochondria and prevents ischemic cardiac remodelling in rats. J Cell Mol Med 2011;15:514-24.

28 Rodondi N, Bauer DC, Cappola AR et al. Subclinical thyroid dysfunction, cardiac function, and the risk of heart failure. The Cardiovascular Health study. J Am Coll Cardiol 2008;52:1152-9.

29 Razvi S, Weaver JU, Butler TJ et al. Levothyroxine treatment of subclinical hypothyroidism, fatal and nonfatal cardiovascular events, and mortality. Arch Intern Med 2012;172:811-7.

30 Monzani F, Caraccio N, Kozàkowà M et al. Effect of levothyroxine replacement on lipid profile and intima-media thickness in subclinical hypothyroidism: a double-blind, placebo-controlled study. J Clin Endocrinol Metab 2004;89:2099-106.

31 Klein I, Ojamaa K. Thyroid hormone and the cardiovascular system. N Engl J Med 2001;344:501-9.

32 Hamilton MA, Stevenson LW, Fonarow GC et al. Safety and hemodynamic effects of intravenous triiodothyronine in advanced congestive heart failure. Am J Cardiol 1998;81:443-7.

33 Moruzzi P, Doria E, Agostoni PG. Medium-term effectiveness of L-thyroxine treatment in idiopathic dilated cardiomyopathy. Am J Med 1996;101:461-7.

34 Pearce SH, Brabant G, Duntas LH et al. 2013 ETA Guideline: Management of Subclinical Hypothyroidism. Eur Thyroid J 2013;2:215-28.

Address for correspondence: Dr S Razvi, Queen Elizabeth Hospital, Gateshead NE9 6SX, UK. Email: salman.razvi@ncl.ac.uk 